\title{
Participating In The Race Across AMerica In A Team Of Eight Cyclists: Do Not Neglect Crew Preparation
}

This article was published in the following Dove Press journal: Open Access Journal of Sports Medicine

\section{Kenny Guex' \\ Emilie Serain' \\ Gerald Gremion ${ }^{2}$ \\ Cyril Besson (1D) ${ }^{2}$ \\ Raphael Faiss ${ }^{3}$ \\ Jocelyne Majo (iD ${ }^{4}$ \\ Francis Degache ${ }^{1,3,5}$}

'School of Health Sciences (HESAV), University of Applied Sciences and Arts Western Switzerland (HES-SO), Lausanne, Switzerland; ${ }^{2}$ Department of Sports Medicine, Centre Hospitalier Universitaire Vaudois, Lausanne, Switzerland; ${ }^{3}$ Institute of Sports Sciences, Faculty of Biology and Medicine, University of Lausanne, Lausanne, Switzerland; ${ }^{4}$ School of Management and Engineering Vaud (HEIG-VD), University of Applied Sciences and Arts Western Switzerland (HES-SO), Delémont, Switzerland; ${ }^{5}$ Therapeutic and Performance Sports Institute, MotionLab, Lausanne, Switzerland
Correspondence: Francis Degache Therapeutic and Performance Sports Institute, MotionLab, Lausanne,

Switzerland

$\mathrm{Tel}+33648951966$

Email fdegache@motion-lab.ch
Background: The Race Across AMerica (RAAM) is considered as one of the longest, and most difficult cycling races in the world. It can be performed in solo or in relay of two, four or eight persons.

Purpose/method: The aim of the present study was to investigate several physiological, perceptual and psychological responses before, during and after RAAM in a team of eight amateur cyclists. Because logistics of all team is demanding and complex, crew members have followed the same testing procedure.

Results: The main result is that parameters were globally not altered to a greater extent in cyclists than in crew members over the course of the RAAM, and that all variables returned to pre-race levels 1 week after the end of the race in both groups. In crew, body fat was decreased ( $p$ $<0.05)$ at mid-race (Mid) vs 1 week before the RAAM (Pre) $(-1.5 \%)$ and total body water was increased $(\mathrm{p}<0.05)$ at Mid vs Pre $(+2.5 \%)$. In pre-relay quadriceps strength in cyclists was significantly lower $(\mathrm{p}<0.05)$ at Mid vs Pre $(41.6 \pm 9.1$ vs $45.0 \pm 11.2 \mathrm{~N}, \mathrm{~d}=0.36)$. Therefore, performing the race with eight well-prepared amateur cyclists seems to decrease potential risks on health. In crew, quadriceps strength remained stable at each assessment time but general fatigue increased all along the race. Anger was increased $(p<0.05)$ at Mid vs Pre in crew.

Conclusion: The most important message of this study is that crew members for a team of eight require adequate and sufficient preparation and training. Roles and responsibilities need to be thoroughly defined; individuals need to know each other beforehand and they must be prepared for sleep deprivation. The results of this study show the importance of the preparation of two teams: crew members, as well as cyclists.

Keywords: ultra-endurance exercise, strength, mood, sleep, body fat, pain

\section{Introduction}

Ultra-endurance cycling races (UECR), defined as competitions exceeding $6 \mathrm{hrs}$ of duration, have become very popular in recent years. ${ }^{1-3}$ The Race Across AMerica (RAAM) is the oldest UECR and is considered as one of the longest, and most difficult in the world. ${ }^{4,5}$ Racers must cycle across the United States of America from West to East in solo or in relay of two, four or eight persons. RAAM represents a unique opportunity to investigate responses of the human body to an extreme load and stress (i.e. distance, elevation, climate). ${ }^{6}$ In the context of a team, it is the occasion to test both the cyclists and crew members. However, to our knowledge, no study has investigated a team of eight competing in this event and its accompanying staff members. 
During multiday UECR, many challenges (e.g. nutrition, hydration, sleep deprivation and environmental conditions) could affect physiological, perceptual, psychological, and performance variables. ${ }^{7-11}$ Previous studies on RAAM demonstrated that a negative energetic balance occurred due to a mean daily energy deficit of about $8500 \mathrm{kcal}$ in solo and $\sim 1500 \mathrm{kcal}$ in a team of four. ${ }^{89}$ In solo, the latter led to a $5 \mathrm{~kg}$ body weight loss, for example. However, a decrease of body weight could also be influenced by changes in hydration status, ${ }^{8} 12$ since it was demonstrated that effective body water changes were correlated to the body mass change in an UECR of $164 \mathrm{~km} .{ }^{12}$ The impact on other physiological parameters, like blood measurement, power output, strength and aerobic capacity have also been studied in UECR. ${ }^{3,6,13}$ While neuromuscular fatigue and strength parameters were well described in ultra-endurance running races, ${ }^{14,15}$ it is not the case in UECR, where only one study showed no significant loss of knee extension strength 5 days after a race of $1700 \mathrm{~km}^{3}{ }^{3}$

Besides, perceptual parameters were explored in nonelite cyclists during an UECR of $164 \mathrm{~km} .{ }^{1}$ An increase of pain, thirst, perceived exertion associated with muscle cramps from the $97 \mathrm{~km}$ was observed. Regarding the amount of sleep, about $\sim 2$ hrs of sleep per day were found in both solo and team of four cyclists during RAAM. ${ }^{8,9}$ To our knowledge, no other study reported perceptual parameters during RAAM.

Perturbations in psychological parameters (e.g. emotions and mood) are also present in UECR. ${ }^{10,11,16}$ For example, during RAAM, cyclists reported that they experienced an optimal emotional state for less than $50 \%$ of the total competition. ${ }^{11}$ This emotional disturbance was associated with poor sleep duration and quality combined with energy deficit. ${ }^{11}$ Dehydration status could also negatively affect the mood with a decreased in vigor. ${ }^{10}$ Consequently, maintaining an optimal emotional state, which is associated to functional performance, represents a challenge for athletes and crew members. ${ }^{11,17}$

The aim of the present study was to investigate several physiological, perceptual and psychological responses before, during and after RAAM in a team of eight amateur cyclists. Because logistics of all team is demanding and complex, and because no previous study has investigated this population, crew members have followed the same testing procedure. It was hypothesised that physiological, perceptual and psychological parameters would be altered to a greater extent in cyclists than in crew members over the course of the RAAM. Moreover, it was hypothesised that all measured variables would return to pre-race levels 1 week after the end of the RAAM.

\section{Methods}

\section{Participants}

Sixteen participants, all cyclists or crew members of a team of eight cyclists participating in the RAAM, took part in the present study. Four females and four males $(34.1 \pm 12.6$ years, $175.7 \pm 9.2 \mathrm{~cm}, 69.5 \pm 10.7 \mathrm{~kg}$ ) composed the cyclist group (Cyclists), while one female and seven males (38.9 \pm 15.9 years, $178.9 \pm 6.8 \mathrm{~cm}, 71.4 \pm 10.2 \mathrm{~kg}$ ) composed the crew members group (Crew). Cyclists included amateur athletes, who followed a supervised and individualised training program in the year before the RAAM with a mean cycling load of $4260 \pm 1420 \mathrm{~km}$. Crew included a crew chief, a physician, a physical therapist, a camera operator, a cycling coach, a bicycle mechanic and two drivers. The entire crew met one time before the race for a 1 day team building event, during which roles and responsibilities of each person, as well as global organisation of the crew were addressed. Prior to the beginning of the study, participants signed an informed consent after explanation of the study protocol, data collection procedures and significance of the study objectives. Ethical approval for the project was obtained from the competent committee on human research (Commission cantonale d'éthique de la recherche sur l'être humain, CER-VD, Agreement 2017-00027, Lausanne, Switzerland) and the protocol was run in accordance with the principles of the Declaration of Helsinki.

\section{Experimental Design}

The race supporting this study was the RAAM, which consists of cycling in one continual stage $\sim 4900 \mathrm{~km}$ from Oceanside, CA to Annapolis, MD with a total positive elevation change of $\sim 50,000 \mathrm{~m}$. Team racers have a maximum of $216 \mathrm{hrs}$ to complete the race. The present study investigated physiological, perceptual and psychological responses in Cyclists and Crew. During the race, the cyclists were split in four duos, each assisted by two crew members. During the race, each duo covered $\sim 1240 \mathrm{~km}$ in eight relays of $\sim 6$ hrs (relay 1: duo 1 - duo 2 - duo 3 - duo 4; relay 2: duo $1-$ duo $2-\ldots$ ). The pacing strategy and repartition of the efforts during each relay were free, with however a trend for each cyclist to cycle three times $\sim 1 \mathrm{hr}$ with $\sim 1 \mathrm{hr}$ rest period between each cycling bout.

Participants were tested four times (Figure 1): 1 week before the RAAM (Pre); at about mid-race (Mid) between 


\begin{tabular}{|c|c|c|c|c|c|c|c|c|c|c|}
\hline Test period & Pre & \multicolumn{4}{|c|}{ Mid } & \multicolumn{4}{|c|}{ End } & Post \\
\hline When & $\begin{array}{l}\text { Post- } \\
\text { sleep }\end{array}$ & $\begin{array}{l}\text { Pre- } \\
\text { relay }\end{array}$ & $\begin{array}{l}\text { Post- } \\
\text { relay }\end{array}$ & $\begin{array}{l}\text { Pre- } \\
\text { sleep }\end{array}$ & $\begin{array}{l}\text { Post- } \\
\text { sleep }\end{array}$ & $\begin{array}{l}\text { Pre- } \\
\text { relay }\end{array}$ & $\begin{array}{l}\text { Post- } \\
\text { relay }\end{array}$ & $\begin{array}{l}\text { Pre- } \\
\text { sleep }\end{array}$ & $\begin{array}{l}\text { Post- } \\
\text { sleep }\end{array}$ & $\begin{array}{l}\text { Post- } \\
\text { sleep }\end{array}$ \\
\hline Where & Home & & & & & & & & & Home \\
\hline
\end{tabular}

\begin{tabular}{|c|c|c|c|c|c|c|c|c|}
\hline \multicolumn{9}{|c|}{ Physiological parameters } \\
\hline $\begin{array}{c}\text { Anthropometric } \\
\text { parameters }\end{array}$ & $\begin{array}{l}\text { Cyclists } \\
+ \text { Crew }\end{array}$ & & & $\begin{array}{l}\text { Cyclists } \\
+ \text { Crew }\end{array}$ & & & $\begin{array}{l}\text { Cyclists } \\
+ \text { Crew }\end{array}$ & $\begin{array}{l}\text { Cyclists } \\
+ \text { Crew }\end{array}$ \\
\hline Quadriceps strength & $\begin{array}{l}\text { Cyclists } \\
+ \text { Crew }\end{array}$ & Cyclists & Cyclists & Crew & Cyclists & Cyclists & Crew & \\
\hline
\end{tabular}

\begin{tabular}{|c|c|c|c|c|c|c|}
\hline \multicolumn{7}{|c|}{ Perceptual parameters } \\
\hline General fatigue & $\begin{array}{l}\text { Cyclists } \\
+ \text { Crew }\end{array}$ & $\begin{array}{l}\text { Cyclists } \\
+ \text { Crew }\end{array}$ & $\begin{array}{l}\text { Cyclists } \\
+ \text { Crew }\end{array}$ & $\begin{array}{l}\text { Cyclists } \\
+ \text { Crew }\end{array}$ & $\begin{array}{l}\text { Cyclists } \\
+ \text { Crew }\end{array}$ & $\begin{array}{l}\text { Cyclists } \\
+ \text { Crew }\end{array}$ \\
\hline Subjective pain & $\begin{array}{l}\text { Cyclists } \\
+ \text { Crew }\end{array}$ & & $\begin{array}{l}\text { Cyclists } \\
+ \text { Crew }\end{array}$ & & $\begin{array}{l}\text { Cyclists } \\
+ \text { Crew }\end{array}$ & $\begin{array}{l}\text { Cyclists } \\
+ \text { Crew }\end{array}$ \\
\hline $\begin{array}{c}\text { Amount and quality } \\
\text { of sleep }\end{array}$ & $\begin{array}{l}\text { Cyclists } \\
+ \text { Crew }\end{array}$ & & $\begin{array}{l}\text { Cyclists } \\
+ \text { Crew }\end{array}$ & & $\begin{array}{l}\text { Cyclists } \\
+ \text { Crew }\end{array}$ & $\begin{array}{l}\text { Cyclists } \\
+ \text { Crew }\end{array}$ \\
\hline
\end{tabular}

Psychological parameters

\begin{tabular}{|c|c|c|c|c|}
\hline Mood states & $\begin{array}{l}\text { Cyclists } \\
+ \text { Crew }\end{array}$ & $\begin{array}{l}\text { Cyclists } \\
+ \text { Crew }\end{array}$ & $\begin{array}{l}\text { Cyclists } \\
+ \text { Crew }\end{array}$ & $\begin{array}{l}\text { Cyclists } \\
+ \text { Crew }\end{array}$ \\
\hline Cohesion of group & $\begin{array}{l}\text { Cyclists } \\
+ \text { Crew }\end{array}$ & $\begin{array}{l}\text { Cyclists } \\
+ \text { Crew }\end{array}$ & $\begin{array}{l}\text { Cyclists } \\
+ \text { Crew }\end{array}$ & $\begin{array}{l}\text { Cyclists } \\
+ \text { Crew }\end{array}$ \\
\hline
\end{tabular}

Figure I Summary of all the measures that were performed in cyclists and crew groups during the study.

Trinidad, CO (km 1930; 23,000 m cumulated positive elevation) and Maize, KS ( $\mathrm{km} 2610 ; \sim 25,000 \mathrm{~m}$ cumulated positive elevation), during the last part of the race (End) between Chillicothe, OH ( $\mathrm{km} \mathrm{4110;} \mathrm{39,000} \mathrm{m} \mathrm{cumulated}$ positive elevation) and Rouzerville, PA (km 4730; 50,000 $m$ cumulated positive elevation); and 1 week after the end of the RAAM (Post). At each assessment time, physiological, perceptual and psychological parameters were tested following the main period of sleep as described below. Moreover, at Mid and End, quadriceps strength was also tested following the last relay (post-relay) of each group of two athletes in Cyclists, while in both groups, general fatigue was also tested before the main period of sleep (pre-sleep).

\section{Physiological Parameters}

Anthropometric parameters (body weight, fat mass and total body water) were acquired when barefoot in underwear using a tetrapolar bioelectrical impedance analysis instrument (Tanita Model BC-601, Tanita Corporation, Tokyo, Japan).

Maximal isometric voluntary quadriceps strength on the right side was tested at $90^{\circ}$ of knee flexion seated using a dynamometer (Baseline Electronic push/pull Dynamometer Model 12-0343, Fabrication Enterprise Inc., NY, USA) mounted on a frame attached to a stable metallic chair. Participants of both groups were asked to push as hard as possible two times 3 swith a $10 \mathrm{~s}$ rest period between both repetitions, while the examiner ensured proper dynamometer positioning on the lower front tibial part. ${ }^{18}$ The best value was kept for calculation. All measures were conducted by the same examiner.

\section{Perceptual Parameters}

Participants of both groups were requested to quantify the level of general fatigue by using a visual analog scale (VAS) with a $10 \mathrm{~cm}$ horizontal line from $0 \mathrm{~cm}=$ no fatigue to $10 \mathrm{~cm}=$ extremely fatigued.

They were asked to quantify the level of subjective pain in four anatomical areas (ankle-leg; knee-thigh; hiplow back; cervical) and digestive feeling by using the VAS from $0 \mathrm{~cm}=$ no pain to $10 \mathrm{~cm}=$ extremely painful.

The amount of sleep in the past $24 \mathrm{hrs}$ was recorded and the quality of sleep was quantified using the VAS from $0 \mathrm{~cm}=$ very bad to $10 \mathrm{~cm}=$ very good.

\section{Psychological Parameters}

To investigate alterations and fluctuations in mood states within Cyclists and Crew, the French version of Profile of Mood State (POMS) questionnaire was used. The POMS comprises 65 single-word mood descriptors, each with a 5point Likert scale anchored by not at all and extremely, assessing one positive mood states (vigor, 8 items), five negative mood states (tension, 9 items; depression, 15 items; anger, 12 items; fatigue, 7 items; confusion, 7 items) and the state of interpersonal relationships (7 
items). An overall global measure of mood (total mood disturbance) was calculated by subtracting vigor from the sum of the five negative mood states. ${ }^{19}$

The cohesion of Cyclists and Crew has been investigated using the Questionnaire sur l'Ambiance du Groupe (QAG), which is a French-language instrument for measuring group cohesion. Cohesion is considered as a multidimensional, dynamic construct composed of the member's perception of the group as a totality and their perceptions of the personal attractiveness of the group. The first dimension is called individual attractions to group (AG) and the second group integration (GI). Each dimension is focused on task (T) and social (S) aspects of the group. The QAG comprises 18 items (AG-T, 4 items; AG-S, 5 items; GI-T, 5 items; GI-S, 4 items), each with a 9-point Likert scale anchored by strongly disagree and strongly agree. Higher scores represented stronger perceptions of cohesiveness. ${ }^{20}$

\section{Statistical Analysis}

Data are expressed as mean \pm standard deviation (SD) in the text and tables, and as mean \pm standard error (SE) in figures. They were screened for normality of distribution and homogeneity of variance using a Shapiro-Wilk normality test and a Barlett's test, respectively. Since normal distribution was not met, Friedman repeated measures analyses of variance on ranks were used to test differences across each assessment time for both groups. Tukey posthoc tests were used to localise the differences between means. The importance of the differences found between each assessment time was assessed through the effect size and Cohen's $d$ coefficient, ${ }^{21}$ interpreted as follows: small difference: $0.20 \leq \mathrm{d}<0.50$, medium difference: $0.50 \leq \mathrm{d}<$ 0.80 , large difference: $0.80 \leq \mathrm{d}<1.30$, and very large difference: $d \geq 1.30$. To examine Cyclists vs Crew differences, Mann-Whitney rank sum test was used. For all statistical analyses, significance was set at $\mathrm{p}<0.05$. Statistical analyses were performed with SigmaPlot 12.5 (Systat Software Inc., San Jose, CA).

\section{Results}

The team of eight cyclists finished the RAAM in 7 days 12 hrs and $46 \operatorname{mins}(25.8 \mathrm{~km} / \mathrm{h})$, in 13 th position out of 15 teams. Each cyclist individually rode between 300 and $840 \mathrm{~km}$, with a total positive elevation change between 2310 and $11,530 \mathrm{~m}$. Figure 2 presents positive elevation change (m) and temperature evolution by section. These data were maximal values of the day for all sections. There was a significant difference of

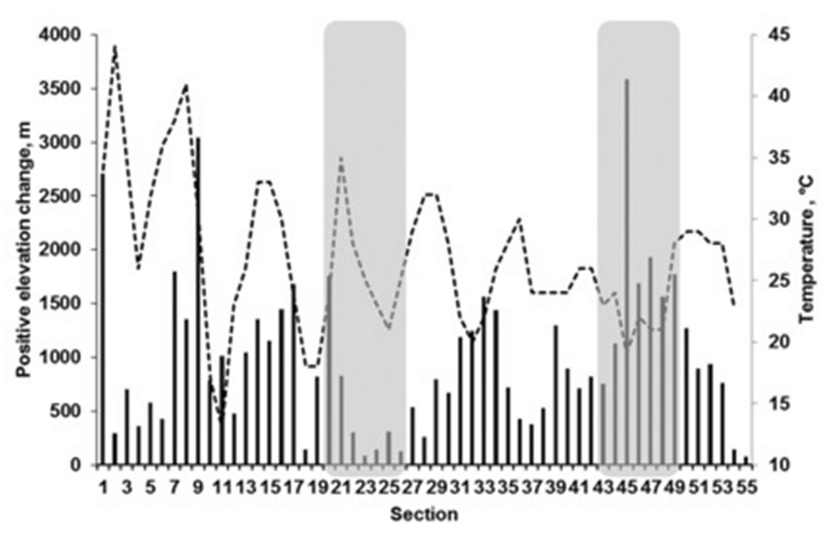

Figure 2 Evolution of positive elevation change $(\mathrm{m})$ (represented by black bar histogram) and maximal temperature $\left({ }^{\circ} \mathrm{C}\right)$ (represented by dashed curve) by section during the race. Left axis represents bar and right grey zones represent the two assessment times during the RAAM.

temperature between the first and the last part of the race $\left(28.6 \pm 7.9\right.$ vs $\left.24.9 \pm 3.6^{\circ} \mathrm{C}, \mathrm{p}<0.05\right)$ but not in elevation change. Due to inadequate participation in physiological, perceptual and psychological measurements, one female in Cyclists and one male in Crew were excluded from the data analysis.

\section{Physiological Parameters}

Body weight, body fat and total body water remained stable at each assessment time in Cyclists. In Crew, body fat was decreased $(\mathrm{p}<0.05)$ at Mid vs Pre $(-1.5 \%)$ and total body water was increased $(\mathrm{p}<0.05)$ at Mid vs Pre $(+2.5 \%)$. No significant difference was observed between groups. Anthropometric parameters in Cyclists and Crew groups are presented in Table 1.

In Cyclists, pre-relay quadriceps strength was lower (p $<0.05)$ at Mid vs Pre $(41.6 \pm 9.1$ vs $45.0 \pm 11.2 \mathrm{~N}, \mathrm{~d}=$ $0.36)$. At Mid, post- vs pre-relay quadriceps strength was decreased $(\mathrm{p}<0.05)$ by $11 \%(37.0 \pm 8.0$ vs $41.6 \pm 9.1 \mathrm{~N}$, $\mathrm{d}=0.58)$. In Crew, quadriceps strength remained stable at each assessment time (Pre, $40.6 \pm 6.1 \mathrm{~N}$; Mid, $39.3 \pm 5.5$ $\mathrm{N}$; End $40.0 \pm 4.4 \mathrm{~N}$ ). No significant difference was observed between groups. For technical reasons, no data were collected at Post. Maximal isometric voluntary quadriceps strength values are shown in Figure 3.

\section{Perceptual Parameters}

In Cyclists, general fatigue was increased $(\mathrm{p}<0.05)$ on VAS at Mid pre-sleep vs Pre $(6.0 \pm 1.3$ vs $1.5 \pm 1.3 \mathrm{~cm}, \mathrm{~d}=3.74)$ and post $(6.0 \pm 1.3 \mathrm{vs} 1.2 \pm 0.6 \mathrm{~cm}, \mathrm{~d}=5.12)$, at Mid postsleep vs. Pre ( $4.2 \pm 0.3$ vs $1.5 \pm 1.3 \mathrm{~cm}, \mathrm{~d}=3.09)$, and at End pre-sleep vs Pre $(5.3 \pm 2.2$ vs $1.5 \pm 1.3 \mathrm{~cm}, \mathrm{~d}=2.27)$. In Crew, 
Table I Anthropometric Parameters

\begin{tabular}{|c|c|c|c|c|c|c|c|c|}
\hline & Pre & Mid & End & Post & $\begin{array}{l}\text { Effect Size } \\
\text { Pre-Mid }\end{array}$ & $\begin{array}{l}\text { Effect Size } \\
\text { Mid-End }\end{array}$ & $\begin{array}{l}\text { Effect Size } \\
\text { End-Post }\end{array}$ & $\begin{array}{l}\text { Effect Size } \\
\text { Pre-Post }\end{array}$ \\
\hline \multicolumn{9}{|c|}{ Body weight, kg } \\
\hline Cyclists & $69.5 \pm 10.7$ & $70.0 \pm 11.8$ & $70.6 \pm 11.9$ & $69.4 \pm 10.8$ & 0.05 & 0.06 & 0.11 & 0.01 \\
\hline Crew & $73.4 \pm 9.5$ & $72.9 \pm 9.0$ & $72.7 \pm 9.1$ & $72.5 \pm 9.4$ & 0.06 & 0.02 & 0.02 & 0.10 \\
\hline \multicolumn{9}{|c|}{ Body fat, \% } \\
\hline Cyclists & $14.0 \pm 6.1$ & $13.7 \pm 5.3$ & $12.9 \pm 6.4$ & $14.0 \pm 6.6$ & 0.06 & 0.15 & 0.18 & 0.00 \\
\hline Crew & $10.5 \pm 3.2$ & $8.0 \pm 3.0 * \$$ & $8.6 \pm 2.8$ & $9.0 \pm 3.4$ & 0.88 (large) & 0.23 (small) & 0.14 & 0.50 (medium) \\
\hline \multicolumn{9}{|c|}{ Total body water, \% } \\
\hline Cyclists & $63.4 \pm 4.4$ & $63.5 \pm 3.5$ & $65.1 \pm 5.9$ & $63.6 \pm 4.8$ & 0.03 & 0.36 (small) & 0.30 (small) & 0.05 \\
\hline Crew & $65.1 \pm 3.0$ & $67.6 \pm 3.4^{*}$ & $66.9 \pm 3.3$ & $66.5 \pm 3.9$ & 0.85 (large) & 0.23 (small) & 0.12 & 0.44 (small) \\
\hline
\end{tabular}

Notes: $*_{p}<0.05$ for difference with Pre; ${ }_{p} p<0.05$ for difference with Cyclists. Data are presented as mean \pm SD.

general fatigue was increased $(\mathrm{p}<0.05)$ at Mid pre-sleep vs Pre $(6.4 \pm 2.3$ vs $1.3 \pm 1.4 \mathrm{~cm}, \mathrm{~d}=2.89)$ and post $(6.4 \pm 2.3 \mathrm{vs}$ $2.1 \pm 1.4 \mathrm{~cm}, \mathrm{~d}=2.44)$, at Mid post-sleep vs Post $(3.9 \pm 2.0 \mathrm{vs}$ $2.1 \pm 1.4 \mathrm{~cm}, \mathrm{~d}=1.13)$, and at End pre-sleep vs Pre $(5.1 \pm 2.0$ vs $1.3 \pm 1.4 \mathrm{~cm}, \mathrm{~d}=2.38)$ and Post $(5.1 \pm 2.0$ vs $2.1 \pm 1.4 \mathrm{~cm}$, $\mathrm{d}=1.88$ ). No significant difference was observed between groups. Ratings of general fatigue are displayed in Figure 4.

At each assessment time, pain in ankle-leg, knee-thigh, hip-low back, and cervical areas and digestive feeling remained below $2 \mathrm{~cm}$ on VAS in both Cyclists and Crew. No significant change between times and groups was observed.

Sleep duration was decreased $(p<0.05)$ at Mid vs Pre in Cyclists $(-33 \%)$ and Crew $(-38 \%)$, and at Mid vs. Post in Cyclists $(-31 \%)$ and Crew $(-44 \%)$. Quality of sleep was decreased $(\mathrm{p}<0.05)$ on VAS at Mid vs Pre $(-3.5 \mathrm{~cm})$ and at Mid vs Post $(-4.2 \mathrm{~cm})$ in Cyclists, while it remained

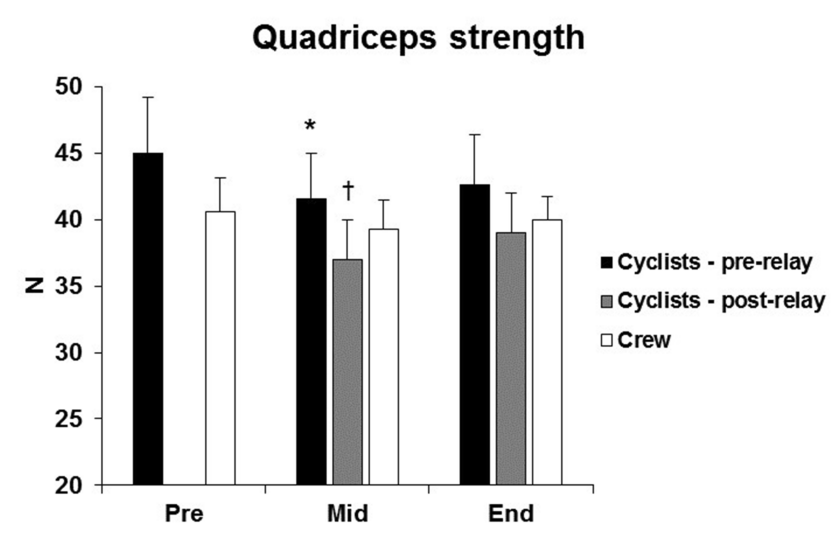

Figure 3 Maximal isometric voluntary quadriceps strength at pre, mid and end in cyclists and crew groups. ${ }^{*} p<0.05$ for difference with Pre; ${ }^{\dagger} p<0.05$ for difference with cyclists pre-relay. Data are presented as mean $\pm \mathrm{SE}$. stable in Crew. There was no significant sleep duration difference between groups. Quality of sleep was worse (p $<0.05)$ in Cyclists vs Crew at Mid. Sleep parameters in Cyclists and Crew are presented in Table 2.

\section{Psychological Parameters}

Tension was decreased $(\mathrm{p}<0.05)$ at Post vs Pre in Cyclists. Anger was increased $(p<0.05)$ at Mid vs Pre in Crew. In both groups, vigor was decreased $(\mathrm{p}<0.05)$ and fatigue was increased $(\mathrm{p}<0.05)$ at Mid vs Pre and at Mid vs Post. Total mood disturbance was increased $(p<0.05)$ at Mid vs End and at Mid vs Post in Cyclists and at Mid vs Pre and at Mid vs Post in Crew. Interpersonal relations were decreased $(p<0.05)$ at Mid vs Post in Crew. No significant difference was observed between groups for the mood aspects, excepted

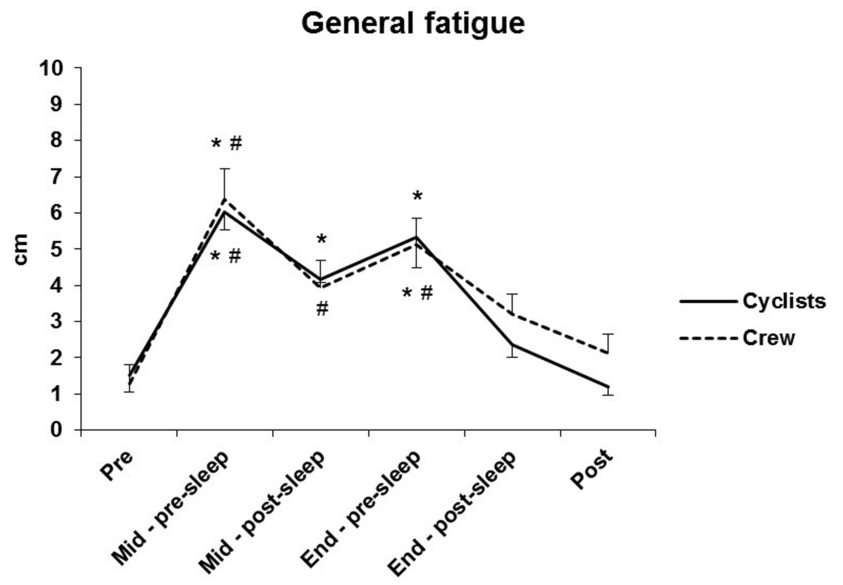

Figure 4 General fatigue at each assessment time in cyclists and crew groups. $*_{p}<$ 0.05 for difference with pre; ${ }^{\#} p<0.05$ for difference with post. Data are presented as mean $\pm \mathrm{SE}$. 
Table 2 Sleep Parameters

\begin{tabular}{|c|c|c|c|c|c|c|c|c|}
\hline & Pre & Mid & End & Post & $\begin{array}{l}\text { Effect Size } \\
\text { Pre-Mid }\end{array}$ & $\begin{array}{l}\text { Effect Size } \\
\text { Mid-End }\end{array}$ & $\begin{array}{l}\text { Effect Size } \\
\text { End-Post }\end{array}$ & $\begin{array}{l}\text { Effect Size } \\
\text { Pre-Post }\end{array}$ \\
\hline \multicolumn{9}{|c|}{ Sleep duration per day, $h$} \\
\hline Cyclists & $7.2 \pm 1.0$ & $4.8 \pm 1.6^{*}$ & $5.6 \pm 1.5$ & $7.0 \pm 1.5^{\#}$ & 1.94 (very large) & 0.56 (medium) & I.0I (large) & 0.17 \\
\hline Crew & $7.3 \pm 0.8$ & $4.5 \pm 1.2^{*}$ & $5.7 \pm 0.9$ & $8.0 \pm 1.2^{\#}$ & 2.97 (very large) & I.22 (large) & 2.34 (very large) & 0.74 (medium) \\
\hline \multicolumn{9}{|c|}{ Quality of sleep, cm } \\
\hline Cyclists & $7.6 \pm 2.7$ & $4.1 \pm 2.5^{*}$ & $7.3 \pm 2.9$ & $8.3 \pm 2.0^{\#}$ & I.45 (very large) & I.28 (large) & 0.43 (small) & 0.32 (small) \\
\hline Crew & $7.9 \pm 1.2$ & $6.6 \pm 2.4^{\$}$ & $7.3 \pm 1.8$ & $8.6 \pm 1.3$ & 0.74 (medium) & 0.36 (small) & 0.89 (large) & 0.60 (medium) \\
\hline
\end{tabular}

Notes: ${ }^{*} p<0.05$ for difference with Pre; ${ }^{*} p<0.05$ for difference with Mid; ${ }^{\$} p<0.05$ for difference with Cyclists. Data are presented as mean \pm SD.

for the interpersonal relationships at Mid, which were lower $(\mathrm{p}<0.05)$ in Crew vs Cyclists. Mood state values in Cyclists and Crew are presented in Table 3.
The four dimensions of group cohesion (social and task aspects of individual attractions to group, and social and task aspects of group integration) remained stable at each

Table 3 Profile Of Mood State

\begin{tabular}{|c|c|c|c|c|c|c|c|c|}
\hline & Pre & Mid & End & Post & $\begin{array}{l}\text { Effect Size } \\
\text { Pre-Mid }\end{array}$ & $\begin{array}{l}\text { Effect Size } \\
\text { Mid-End }\end{array}$ & $\begin{array}{l}\text { Effect Size } \\
\text { End-Post }\end{array}$ & $\begin{array}{l}\text { Effect Size } \\
\text { Pre-Post }\end{array}$ \\
\hline \multicolumn{9}{|c|}{ Tension } \\
\hline Cyclists & $7.4 \pm 3.7$ & $4.3 \pm 2.9$ & $3.6 \pm 1.7$ & $3.6 \pm 1.5^{*}$ & 1.01 (large) & 0.32 (small) & 0.00 & I.45 (very large) \\
\hline Crew & $6.4 \pm 4.0$ & $4.9 \pm 3.2$ & $6.3 \pm 3.4$ & $4.7 \pm 2.1$ & 0.45 (small) & 0.46 (small) & 0.61 (medium) & 0.58 (medium) \\
\hline \multicolumn{9}{|c|}{ Depression } \\
\hline Cyclists & $2.4 \pm 2.4$ & $2.7 \pm 4.4$ & $1.0 \pm 2.2$ & $2.0 \pm 3.2$ & 0.09 & 0.53 (medium) & 0.39 (small) & 0.15 \\
\hline Crew & $1.0 \pm 2.2$ & $1.4 \pm 2.8$ & $1.7 \pm 1.7$ & $0.9 \pm 1.6$ & 0.17 & 0.14 & 0.52 (medium) & 0.06 \\
\hline \multicolumn{9}{|l|}{ Anger } \\
\hline Cyclists & $5.9 \pm 4.0$ & $6.9 \pm 5.5$ & $4.7 \pm 1.7$ & $5.9 \pm 4.2$ & 0.23 (small) & 0.58 (medium) & $0.4 \mathrm{I}$ (small) & 0.00 \\
\hline Crew & $3.4 \pm 3.7$ & $8.3 \pm 6.3 *$ & $6.4 \pm 3.9$ & $4.9 \pm 4.9$ & 1.02 (large) & 0.39 (small) & 0.37 (small) & 0.37 (small) \\
\hline \multicolumn{9}{|l|}{ Vigor } \\
\hline Cyclists & $24.4 \pm 3.7$ & $17.7 \pm 6.2^{*}$ & $22.7 \pm 4.2$ & $24.3 \pm 2.9^{\#}$ & I.42 (very large) & 1.02 (large) & 0.48 (small) & 0.03 \\
\hline Crew & $23.1 \pm 5.7$ & $17.3 \pm 5.6 *$ & $18.4 \pm 7.0$ & $21.6 \pm 8.0$ & I.II (large) & 0.19 & 0.46 (small) & 0.23 (small) \\
\hline \multicolumn{9}{|l|}{ Fatigue } \\
\hline Cyclists & $4.1 \pm 1.6$ & $7.6 \pm 1.3^{*}$ & $5.0 \pm 2.8$ & $3.4 \pm 2.1^{\#}$ & 2.59 (very large) & 1.29 (large) & 0.70 (medium) & 0.41 (small) \\
\hline Crew & $3.6 \pm 1.9$ & $7.3 \pm 2.7^{*}$ & $7.3 \pm 3.5$ & $3.3 \pm 2.2^{\#}$ & I.7I (very large) & 0.00 & I. 48 (very large) & 0.16 \\
\hline \multicolumn{9}{|c|}{ Confusion } \\
\hline Cyclists & $6.4 \pm 5.2$ & $6.9 \pm 5.2$ & $4.4 \pm 1.0$ & $4.9 \pm 1.2$ & 0.10 & 0.72 (medium) & 0.49 (small) & 0.42 (small) \\
\hline Crew & $4.7 \pm 2.4$ & $5.4 \pm 2.6$ & $4.3 \pm 3.4$ & $5.0 \pm 2.9$ & 0.30 (small) & 0.39 (small) & 0.24 (small) & 0.12 \\
\hline \multicolumn{9}{|c|}{ Total mood disturbance } \\
\hline Cyclists & $1.9 \pm 14.9$ & $10.6 \pm 22.1$ & $-4.0 \pm 5.9^{\#}$ & $-4.6 \pm 11.5^{\#}$ & 0.50 (medium) & 0.98 (large) & 0.07 & 0.53 (medium) \\
\hline Crew & $-4.0 \pm 14.3$ & $10.3 \pm 16.2 *$ & $7.6 \pm 16.4$ & $-2.9 \pm 13.3^{\#}$ & I.0I (large) & 0.18 & 0.76 (medium) & 0.09 \\
\hline \multicolumn{9}{|c|}{ Interpersonal relationships } \\
\hline Cyclists & $23.3 \pm 4.1$ & $23.3 \pm 4.6$ & $25.1 \pm 2.8$ & $25.4 \pm 2.6$ & 0.00 & $0.5 \mathrm{I}$ (medium) & 0.12 & 0.66 (medium) \\
\hline Crew & $21.6 \pm 5.5$ & $17.1 \pm 5.0^{\$}$ & $20.7 \pm 4.4$ & $22.4 \pm 4.3^{\#}$ & 0.93 (large) & 0.83 (large) & 0.42 (small) & 0.18 \\
\hline
\end{tabular}

Notes: ${ }^{*} p<0.05$ for difference with Pre; ${ }^{*} p<0.05$ for difference with Mid; ${ }^{\$} p<0.05$ for difference with Cyclists. Data are presented as mean \pm SD. 
assessment time in both groups. No significant difference was observed between groups for social and task aspects of individual attractions to group. Social and task aspects of group integration at Pre were lower $(\mathrm{p}<0.05)$ in Crew vs Cyclists $(11.3 \pm 5.8$ vs $18.9 \pm 4.4$ and $27.9 \pm 12.0$ vs $37.4 \pm 2.4$, respectively).

\section{Discussion}

The purpose of the present study was to investigate physiological, perceptual and psychological responses before, during and after the RAAM in cyclists and crew members of a team of eight amateur cyclists. The main result, in contrast to our hypothesis, is that physiological, perceptual and psychological parameters were not altered to a greater extent in cyclists than in crew members (excepted for quadriceps strength and quality of sleep) over the course of the RAAM. However, in line with our hypothesis, all variables returned to pre-race levels (excepted tension in Cyclists, which was largely decreased) 1 week after the end of the race in both groups.

\section{Physiological Parameters}

Globally, anthropometric parameters remained stable in both groups during the race performed in team of eight, whereas important daily energy deficits were reported in solo and team of four leading to a five $\mathrm{kg}$ body weight loss in solo. ${ }^{8,9}$ This result shows that, contrarily to solo and team of four cyclists, it is possible to keep a stable energy balance if participating in a team of eight.

As it could be expected, quadriceps strength was not altered in Crew, but following a relay (usually 3 times $\sim 1 \mathrm{hr}$ with $\sim 1 \mathrm{hr}$ rest period), a loss of $11 \%$ and $8 \%$ of quadriceps strength was found in Cyclists at Mid and End, respectively. This is in line with the $13 \%$ reduction in quadriceps peak torque found after 2 hrs cycling exercise at $65 \%$ of $\mathrm{VO}_{2 \max }{ }^{22}$ These losses could be attributed to both reduced neural input to the muscles and a failure of peripheral contractile mechanisms, but probably not to a decrease in muscle mass, since this last was found to remain stable following an UECR of $600 \mathrm{~km}^{2}$ In Cyclists, pre-relay quadriceps strength was lower $(\mathrm{p}<0.05)$ at Mid vs Pre $(41.6 \pm 9.1$ vs $45.0 \pm 11.2 \mathrm{~N}, \mathrm{~d}=0.36)$. This result could be explained by the fact that in a long-distance race (ultramarathon, ultra-trail, ultra cycling race) we observed a specific pacing strategy. Paradoxically, such extreme exercise seems to induce a relative muscle preservation process due likely to a protective anticipatory pacing strategy during the first half of race. ${ }^{14}$ For technical reasons, no strength data were collected at Post. However, one may think that it would not be different from Pre as previously shown after an UECR of $1700 \mathrm{~km}^{3}$

\section{Perceptual Parameters}

General fatigue followed the same pattern in both groups and appears to be higher at Mid than at End. This larger rise following the first part of the race could be attributed to different factors. In cyclists, total positive elevation was about the same between the two parts of the race. However, the mean temperature was significantly higher during the first part of the race $\left(28.6 \pm 7.9\right.$ vs $\left.24.9 \pm 3.6^{\circ} \mathrm{C}\right)$ (Figure 2). It was shown that heat stress could induce a significant decrease of $6.5 \%$ in cycling performance and increase fatigue perception. ${ }^{23}$ Since crew members spent most of their time in cars with conditioned air, other factors must explain their important fatigue at Mid. For example, it was the first RAAM for all participants and crew members did not undergo any specific training before the race. Moreover, roles and responsibilities of each person were not completely clear during the first days of the race. Consequently, a crisis meeting had to be organised at Mid in order to clarify respective tasks and roles of crew members and to improve global organisation. This could probably explain difficulties of the crew at the beginning of the race causing additional fatigue.

No major pain issue was observed during the study. This result shows that performing the RAAM in team of eight well-trained amateur cyclists would seem to decrease the risks of deteriorating health.

In cyclists and crew, sleep duration and quality mostly decreased at Mid. This observation could also explain the general fatigue higher at Mid than at End. However, sleep duration remained higher than the $2 \mathrm{hrs}$ per day found in both solo and team of four cyclists. ${ }^{8,9,11}$ Sleep deprivation is a classical aspect in ultra-distance competitions. In running, for example, sleep deprivation associated with fatigue was shown to induce a decrease of postural control. ${ }^{24}$ The latter was also shown to be worst during night time, ${ }^{25}$ increasing the risk of accidents. In crew members, sleep deprivation could induce an altered driving ability. This highlights the importance of being prepared to sleep deprivation in both cyclists and crew members before the race.

\section{Psychological Parameters}

Only fatigue and vigor subscales significantly decreased and increased, respectively in both groups at Mid. The pattern of these two parameters appears to be in line 
with general fatigue. In Cyclists, the other emotional states remained relatively stable during the race, while in team of four, an optimal emotional state was reported for less than $50 \%$ of the race. ${ }^{11}$ The long recovery between relays $(\sim 16$ hrs) and the lower sleep deprivation observed in our team could explain this difference. Anger was increased at Mid in Crew, while interpersonal relationships were significantly lower than in cyclists. As previously discussed, the lack of experience and organisation could explain these states. In fact, crew members were not prepared for the conditions of the race, while cyclists were trained for their single cycling task. Initial information and preparation seem to be essential to follow a cyclist group along the RAAM, essentially to ensure logistical aspects. Another set of hypotheses could be related to sharing the same values and goals between individuals (performing or participating?).

This important observation about crew preparation is supported by social and task aspects of group integration and motivation ${ }^{26}$ that were lower in Crew than in Cyclists at Pre. These results highlight the fact that crew members did not sufficiently know each other before the race and that their respective roles were not enough described. Indeed, while cyclists trained during a whole year, the entire crew team met only one time to train before the RAAM.

\section{Study Limitations}

There are some limitations to the present study. The first one is the small sample size. However, other studies about the RAAM have used smaller sample size. ${ }^{8,9,11}$ The second limitation concerns the assessment times during the race. At Mid and End, data were recorded over a window of 600-700 km. Consequently, participants were not tested in the exact same conditions. Thus, results must be considered with caution. Finally, in line with the aim of the study, data analysis was performed on Cyclists and Crew groups, which may have occluded several differences that could have been observed due to gender, different distances covered or climatic factors.

\section{Conclusion}

To our knowledge, this is the first study investigating a team of eight cyclists and its crew during the RAAM, leading to two relevant information. First, performing the race with eight well-prepared amateur cyclists does not present any health-related issue, since the demand on cyclists appears to be lower than in solo and in team of four. Indeed, a single week of recovery was enough for physiological and psychological variables to return to baseline. Second, crew members for a team of eight require adequate and sufficient preparation and training. Role and responsibilities need to be thoroughly defined; individuals need to know each other beforehand and they must be prepared to sleep deprivation. We can argue that in solo, team of two and four a large part of the demand is on the cyclist. In team of eight, with considerable logistical aspects, one may think that it is more fifty-fifty between cyclists and crew members. The results of this study show the importance of the preparation of two teams: crew members, as well as cyclists.

\section{Disclosure}

The authors report no conflicts of interest in this work.

\section{References}

1. Armstrong LE, Casa DJ, Emmanuel H, et al. Nutritional, physiological, and perceptual responses during a summer ultraendurance cycling event. $J$ Strength Cond Res. 2012;26(2):307-318. doi:10.1519/JSC.0b013e318240f677

2. Knechtle B, Wirth A, Knechtle P, Rosemann T. An ultra-cycling race leads to no decrease in skeletal muscle mass. Int J Sports Med. 2009;30(3):163-167. doi:10.1055/s-0028-1104585

3. Clemente-Suarez VJ. Changes in biochemical, strength, flexibility, and aerobic capacity parameters after a $1700 \mathrm{~km}$ ultraendurance cycling race. Biomed Res Int. 2014;2014:602620. doi:10.1155/2014/ 602620

4. Shoak MA, Knechtle B, Knechtle P, Rust CA, Rosemann T, Lepers R. Participation and performance trends in ultracycling. Open Access J Sports Med. 2013;4:41-51. doi:10.2147/OAJSM.S40142

5. Zingg M, Knechtle B, Rust CA, Rosemann T, Lepers R. Age and gender difference in non-drafting ultra-endurance cycling performance - the 'Swiss Cycling Marathon'. Extrem Physiol Med. 2013;2(1):18. doi:10.1186/2046-7648-2-18

6. Kupchak BR, Kazman JB, Vingren JL, et al. Blood hemostatic changes during an ultraendurance road cycling event in a hot environment. Wilderness Environ Med. 2017;28(3):197-206. doi:10.1016/ j.wem.2017.05.002

7. Bescos R, Rodriguez FA, Iglesias X, et al. Physiological demands of cyclists during an ultra-endurance relay race: a field study report. Chin J Physiol. 2011;54(5):339-346.

8. Knechtle B, Enggist A, Jehle T. Energy turnover at the Race Across AMerica (RAAM) - a case report. Int J Sports Med. 2005;26(6):499503. doi: $10.1055 / \mathrm{s}-2004-821136$

9. Hulton AT, Lahart I, Williams KL, et al. Energy expenditure in the Race Across America (RAAM). Int J Sports Med. 2010;31(7):463467. doi: $10.1055 / \mathrm{s}-0030-1251992$

10. Moyen NE, Ganio MS, Wiersma LD, et al. Hydration status affects mood state and pain sensation during ultra-endurance cycling. $J$ Sports Sci. 2015;33(18):1962-1969. doi:10.1080/02640414.2015.1021275

11. Lahart IM, Lane AM, Hulton A, et al. Challenges in maintaining emotion regulation in a sleep and energy deprived state induced by the $4800 \mathrm{Km}$ ultra-endurance bicycle race; The Race Across AMerica (RAAM). J Sports Sci Med. 2013;12(3):481-488.

12. Armstrong LE, Johnson EC, Ganio MS, et al. Effective body water and body mass changes during summer ultra-endurance road cycling. $J$ Sports Sci. 2015;33(2):125-135. doi:10.1080/02640414.2014.932918 
13. Schumacher YO, Ahlgrim C, Prettin S, Pottgiesser T. Physiology, power output, and racing strategy of a Race Across America finisher. Med Sci Sports Exerc. 2011;43(5):885-889. doi:10.1249/MSS.0b013e3181fec009

14. Saugy J, Place N, Millet GY, et al. Alterations of neuromuscular function after the world's most challenging mountain ultra-marathon. PLoS One. 2013;8(6):e65596. doi:10.1371/journal.pone.0065596

15. Millet G, Tomazin K, Verges S, et al. Neuromuscular consequences of an extreme mountain ultra-marathon. PLoS One. 2011;6(2): e17059. doi:10.1371/journal.pone.0017059

16. Lane AM, Wilson M. Emotions and trait emotional intelligence among ultra-endurance runners. J Sci Med Sport. 2011;14(4):358362. doi:10.1016/j.jsams.2011.03.001

17. Lane AM, Terry PC, Stevens MJ, Barney S, Dinsdale SL. Mood responses to athletic performance in extreme environments. J Sports Sci. 2004;22 (10):886-897. discussion 897. doi:10.1080/02640410400005875

18. Katoh M. Reliability of isometric knee extension muscle strength measurements made by a hand-held dynamometer and a belt: a comparison of two types of device. J Phys Ther Sci. 2015;27 (3):851-854. doi:10.1589/jpts.27.851

19. McNair DM, Lorr M, Droppleman LF. Revised Manual for the Profile of Mood States. San Diego, CA: Educational and Industrial Testing Services; 1992.
20. Heuze JP, Fontayne P. Questionnaire sur l'Ambiance du groupe: a french-language instrument for measuring group cohesion. J Sport Exercise Psy. 2002;24(1):42-67. doi:10.1123/jsep.24.1.42

21. Cohen J. Statistical Power Analysis for the Behavioral Sciences. 2nd ed. Hillsdale, NJ: Lawrence Erlbaum Associates; 1988.

22. Lepers R, Hausswirth C, Maffiuletti N, Brisswalter J, van Hoecke J. Evidence of neuromuscular fatigue after prolonged cycling exercise. Med Sci Sports Exerc. 2000;32(11):1880-1886. doi:10.1097/ 00005768-200011000-00010

23. Tatterson AJ, Hahn AG, Martin DT, Febbraio MA. Effects of heat stress on physiological responses and exercise performance in elite cyclists. J Sci Med Sport. 2000;3(2):186-193.

24. Degache F, Van Zaen J, Oehen L, et al. Alterations in postural control during the world's most challenging mountain ultra-marathon. PLoS One. 2014;9(1):e84554. doi:10.1371/journal.pone.0084554

25. Bougard C, Lepelley MC, Davenne D. The influences of time-of-day and sleep deprivation on postural control. Exp Brain Res. 2011;209 (1):109-115. doi:10.1007/s00221-010-2524-8

26. Krouse RZ, Ransdell LB, Lucas SM, Pritchard ME. Motivation, goal orientation, coaching, and training habits of women ultrarunners. $J$ Strength Cond Res. 2011;25(10):2835-2842. doi:10.1519/JSC.0b01 3 e318204caa0

\section{Publish your work in this journal}

Open Access Journal of Sports Medicine is an international, peerreviewed, open access journal publishing original research, reports, reviews and commentaries on all areas of sports medicine. The manuscript management system is completely online and includes a very quick and fair peer-review system. Visit http://www.dovepress com/testimonials.php to read real quotes from published authors. 